\title{
METODOLOGÍA CUALITATIVA PARA LA VALIDACIÓN DE UN CUES- TIONARIO DE RECURSOS Y SERVICIOS EN CUIDADOS PALIATIVOS
}

\author{
QUALITATIVE METHODOLOGY FOR A PALLIATIVE CARE RESOURCES AND SERVICES \\ QUESTIONNAIRE VALIDATION
}

\begin{abstract}
Fátima Izquierdo-Botica', Diego Ruiz-López², Mercedes Guerra-Rodríguez', María Teresa García-Baquero Merino ${ }^{2}$ y Juan Antonio Blasco-Amaro'

1 Unidad de Evaluación de Tecnologías Sanitarias (UETS), Agencia Laín Entralgo. Consejería de Sanidad, Comunidad de Madrid.

${ }^{2}$ Coordinación Regional de Cuidados Paliativos. Consejería de Sanidad, Comunidad de Madrid.
\end{abstract}

Resumen

Objetivo: Establecer la validez de composición, contenido y criterios de un cuestionario de recursos para la provisión de servicios en Cuidados Paliativos (CP) a través de una metodología cualitativa, mediante grupos focales con profesionales de CP; en el marco de un proyecto de validación y evaluación de los resultados de un cuestionario de recursos para la provisión de servicios en (CP) en la Comunidad de Madrid (CM).

Método: Se lleva a cabo un abordaje cualitativo para la validación del cuestionario de recursos. Se selecciona el grupo de discusión como técnica de elección para obtener un discurso en base a la experiencia personal de los profesionales implicados en el ámbito de los CP. Los grupos se componen de médicos, enfermeras, psicólogos, trabajadores sociales y agente de pastoral.

Resultado: Los temas de discusión en los grupos fueron: dispersión geográfica de los recursos humanos, motivación laboral y personal, "ayuda psico-emocional" para profesionales, valoración integral y holística del paciente, equidad de los equipos, presupuesto social, apoyo al cuidador, ayuda entre equipos, sobrecarga laboral y humana, relación con la gerencia, futuro de los equipos: "estar quemados", trabajo en equipo, formación en CP, labor de

\section{Abstract}

Objective: To establish the composition, content and criteria validity of a questionnaire of resources for services provision in Palliative Care (PC) through a qualitative methodology using focus groups with PC professionals. This is a part of a validation and evaluation research project for the provision of services $(\mathrm{CP})$ in the Community of Madrid (CM).

Methods: We performed a qualitative approach to the resource questionnaire validation. It was selected the focus group technique based on the personal experience of the professionals involved in PC. The groups were made up of physicians, nurses, psychologists, social workers and pastoral agent.

Results: Discussion items in the groups were: human resources, geographical dispersion, work and personal motivation, "psycho-emotional" help for professionals, comprehensive and holistic patient approach, equipment equity, social budget, caregiver support, support between teams, overburden and human relationship with CEOs, future of teams: "be burned", team-working, teaching in PC, PC nursing work, PC welcome protocol and terminological and conceptual clarifications.

Conclusions: This study tries to be the starting point for the development and validation of the PC provision of services

\section{Correspondencia:}

Red de Investigación de Cuidados Paliativos de la Comunidad de Madrid.

Coordinación Regional de Cuidados Paliativos de la Comunidad de Madrid.

Plaza Carlos Trías Beltrán 7. Edicio Sollube - 28020 Madrid.

Email: crcp@salud.madrid.org 
enfermería en $\mathrm{CP}$, protocolo de acogida en $\mathrm{CP}$ y aclaraciones terminológicas o conceptuales.

Conclusiones: Este trabajo pretende ser el punto de partida para la elaboración y validación de un cuestionario en la provisión de servicios en $C P$, y así evaluar los recursos humanos y técnicos de nuestra red asistencial sanitaria paliativa.

Palabras clave: Cuidados Paliativos, Investigación cualitativa, Grupos Focales, Evaluación de Programa. questionnaire. It will be useful to assess the human and technical resources of our palliative health care network.

Key words: Palliative Care, Qualitative Research, Focus Groups, Program Evaluation.

\section{INTRODUCCIÓN}

En el marco de un proyecto de validación y evaluación de los resultados de un cuestionario de recursos para la provisión de servicios en Cuidados Paliativos $(\mathrm{CP})^{(1,2)}$ se desarrolló una fase previa de revisión sistemática de la literatura sobre recursos y métodos de evaluación de unidades y servicios de $\mathrm{CP}$, a nivel nacional e internacional. Seguidamente, se llevó a cabo una fase con metodología cualitativa, basada en grupos de discusión con profesionales pertenecientes a las unidades de CP domiciliarias y hospitalarias de la Comunidad de Madrid (CM).

El cuestionario de recursos lo componen 70 ítems cerrados y un análisis tipo DAFO; su objetivo era evaluar la dotación técnica y humana de los servicios de CP. En una etapa anterior del proyecto, desde noviembre de 2008 hasta marzo de 2009, se contestaron un total de 40 cuestionarios por parte de profesionales específicos de las unidades de $\mathrm{CP}$, tanto domicilio como en los hospitales, de la CM. Los datos fueron recogidos por la Coordinación Regional de $\mathrm{CP}$ de la CM siguiendo un formato de entrevistas grupales con los equipos de CP.

El inicio de la validación del cuestionario de recursos comienza con la comparación de dicho cuestionario con los recursos y herramientas identificados en la revisión sistemática.
El objetivo de nuestro estudio es establecer la validez de composición, contenido y criterios de un cuestionario de recursos para la provisión de servicios en Cuidados Paliativos ${ }^{(1,2)}$ a través de una metodología cualitativa, mediante grupos focales con profesionales de CP.

\section{MÉTODO}

Se lleva a cabo un abordaje cualitativo, centrado en el análisis de consistencia interna, estabilidad temporal y concordancia, que contribuye a la validación del cuestionario de recursos. Para ello, se selecciona el grupo de discusión como técnica de elección; siguiendo un guión de preguntas previamente elaboradas ad hoc se espera obtener un discurso en base a la experiencia personal de los profesionales implicados en el ámbito de los CP. Los grupos se componen de médicos/as, enfermeros/as, psicólogos/as, trabajadores sociales y agente de pastoral.

El diseño de la investigación cualitativa se realiza siguiendo las fases propuestas por Valles ${ }^{(3)}$ (ver figura 1).

\section{Preparación:}

Para la selección de los profesionales participantes se tienen en cuenta diferentes áreas geográficas y de salud de la CM, 
Figura 1. Fases y tareas del diseño de estudio cualitativo (Esquema modificado de Valles 1999)(3).

\begin{tabular}{|c|c|c|}
\hline \multicolumn{3}{|c|}{$\begin{array}{l}\text { Diseño de estudio cualitativo } 0 \\
\text { cualitativo-cuantitativo }\end{array}$} \\
\hline \multicolumn{3}{|c|}{ Fases y tareas: decisiones de diseño } \\
\hline $\begin{array}{l}\text { Al principio del estudio } \\
\text { Etapa de reflexión y preparación } \\
\text { del proyecto } \\
\text { Tareas: } \\
\text { - De formulación del problema. } \\
\text { - De selección de estrategia } \\
\text { metodológica. } \\
\text { - De selección de casos, contextos, } \\
\text { fechas. }\end{array}$ & $\begin{array}{l}\text { Durante el estudio } \\
\text { Etapa de entrada y realización del } \\
\text { campo } \\
\text { Tareas: } \\
\text { - De gestión (cartas y visitas de } \\
\text { presentación...). } \\
\text { - De ajuste en las técnicas de } \\
\text { recogida. } \\
\text { - De ejecución del campo. } \\
\text { - De archivo y análisis preliminar. }\end{array}$ & $\begin{array}{l}\text { Al final } \\
\text { Etapa de salida, análisis final y } \\
\text { escritura } \\
\text { Tareas: } \\
\text { - De finalización o interrupción del } \\
\text { campo. } \\
\text { - De análisis intenso final. } \\
\text { - De redacción y presentación del } \\
\text { informe. }\end{array}$ \\
\hline & Realidad social & \\
\hline
\end{tabular}

Figura 2. Casillero tipológico con la combinación de los criterios de selección para los grupos de discusión.

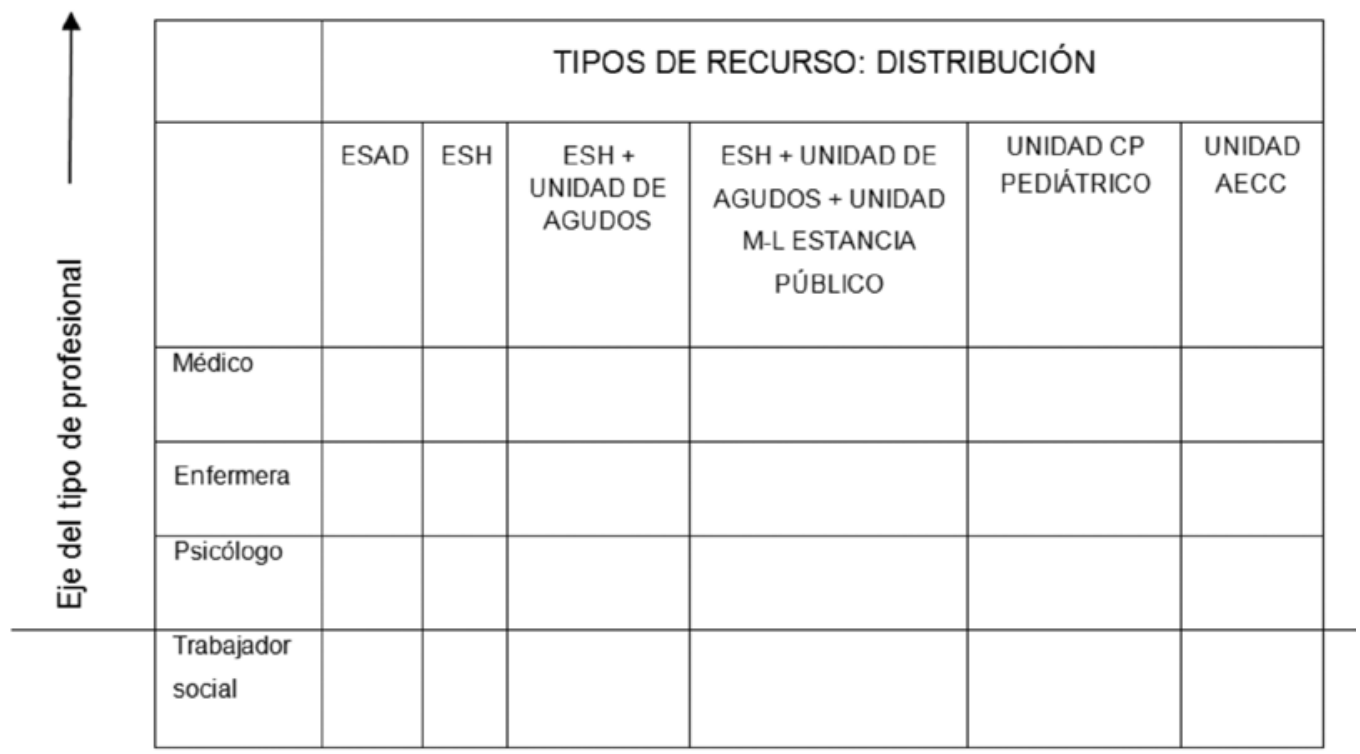


respetando los criterios de selección de heterogeneidad, accesibilidad y representación. Los criterios de muestreo se fundamentan en las 4 preguntas criterio de Gorden para la selección de contextos y casos.

En un casillero tipológico se representa la combinación de los criterios de selección para los grupos de discusión (figura 2).

\section{Trabajo de campo:}

El reclutamiento de los profesionales se hace a través de una persona que cuenta con amplia experiencia en el ámbito de CP y utiliza sus redes profesionales. De este modo, se contacta con 10 profesionales telefónicamente (04/03/2011), con el fin de realizar el primer grupo de discusión y se les envía por correo electrónico (09/03/2011) una breve presentación del proyecto. Del total de los profesionales, finalmente confirmaron asistencia 8, con los que se desarrolló el primer grupo de discusión en la fecha y circunstancias convenidas.

Con el propósito de abarcar un amplio abanico de discursos tipológicamente relevantes, el grupo I se compone de los siguientes profesionales: 3 médicos de Equipos de Soporte Paliativo Domiciliario de 3 sectores distintos de la CM, 1 enfermero de Equipo de Soporte Paliativo Hospitalario, 1 enfermera de Equipo de Soporte Paliativo Domiciliario, 1 psicóloga de Unidad de Camas Concertada de Media y Larga Estancia, 1 psicóloga de Unidad de Cuidados Paliativos Pediátricos y un trabajador social de Unidad de Camas Concertada de Media y Larga Estancia.

Adicionalmente, se establece un segundo grupo de discusión de 4 participantes, en el cual se incluye otro perfil relevante no incluido en el grupo anterior: la figura del agente pastoral. El grupo II se forma con los siguientes profesionales: 1 médico de Unidad de Camas Concertada de Media y Larga Estancia, 1 psicólogo de Unidad de Camas Concertada de Media y Larga Estancia, 1 trabajadora social de Unidad de Cuidados Paliativos Pediátricos y 1 agente pastoral de Unidad de Camas Concertada de Media y Larga Estancia.

Las sesiones se desarrollan en una sala de reuniones de la Agencia Laín Entralgo de Madrid, con una duración estimada de 2 horas y media. Están dirigidas por un moderador y un observador, miembros de la Unidad de Evaluación de Tecnologías Sanitarias (UETS) de dicha agencia. Al inicio de cada reunión, se solicita a los participantes el consentimiento para que la información recabada pueda ser utilizada para los fines que se persiguen en la presente investigación cualitativa. Las experiencias y puntos de vista que exponen los profesionales se recogen en audio con una grabadora.

El grupo de discusión se establece como una reunión con modalidad de entrevista grupal abierta. Con el fin de estructurar el tiempo total disponible se elabora un guión orientado a responder las temáticas de nuestro interés; el guión está compuesto por unas líneas de indagación basadas en los ítems del cuestionario de recursos para la provisión de servicios en CP. Estas líneas o temáticas-estímulo, se muestran en la figura 3.

En base al documento de audio obtenido, se inicia el proceso de transcripción de los grupos de discusión. En esta fase de la investigación los verbatim más representativos son insertados dentro de una categorización básica. De igual modo, se cumplimenta una ficha técnica, en la cual se chequean diversos elementos presentes en el grupo desde la evaluación del observador, y otra segunda ficha, en la que se adjunta la transcripción tras la fase de análisis.

\section{RESULTADOS}

Los resultados obtenidos a través de la fase de análisis de los grupos de discusión 


\section{Figura 3. Líneas de indagación propuestas para los grupos focales, basadas en los ítems del Cuestionario de Recursos para la provisión de servicios en CP.}

1. Recursos técnicos:

1.1. Nombre, tipo y localización de recursos (ESAD, ESH, ESH+ Unidad de Camas Agudas, ESH + Unidad de Camas Agudas+ M-L estancia, CP Pediátricos, Unidad AECC)

1.2. Estancia media del paciente, origen, proceso de derivación

1.3. $\mathrm{N}^{\mathrm{o}}$ de camas

1.4. Historia clínica electrónica

1.5. pacientes oncológicos/no oncológicos

1.6. Móvil/Busca

1.7. Despacho propio

1.8. Sala para familiares

1.9. Conductor/vehículo propio

1.10. Herramientas utilizadas para recoger información del funcionamiento de los servicios (cuestionarios, escalas, indicadores, etc.)

1.11. Evaluación de los costes de los servicios

1.12. Evaluación de los resultados en cuidados

1.13. Evaluación de la satisfacción de los usuarios, familiares y profesionales

1.14. Evaluación de la calidad de vida en salud

1.15. Evaluación de nivel de ansiedad

1.16. Evaluación de demandas, reclamaciones, etc.

1.17. Manejo de GPC

\section{Recursos humanos:}

2.1. Profesionales integrados en el equipo

2.2. Atención domiciliaria

2.3. Cuidados Paliativos pediátricos

2.4. Atención religiosa

2.5. Voluntariado

2.6. Pacientes: interacción con otros profesionales

2.7. Manejo del Dolor: analgésicos/ tratamientos como morfina, etc.

2.8. Formación/reciclaje de los profesionales

2.9. Actividad asistencial

2.10. Docencia

2.11. Investigación

2.12. Necesidades de los profesionales en CP

2.13. Déficit de coordinación entre profesionales. Posibilidades de mejora.

2.14. En relación a su experiencia, adecuación de los recursos que les rodean: Recursos que se utilizan, recursos que faltan, recursos que sobran, etc.

nos llevan a agrupar los datos en los apartados temáticos que figuran en la tabla 1.

En el grupo de discusión II, y con respecto al grupo de discusión I, encontramos temas similares, que saturan el discurso en torno a los recursos materiales y personales con los que cuentan los profesionales de Cuidados Paliativos de la CM. Sobre todo son tres los epígrafes más redundantes: Valoración/Atención integral del paciente en 
Tabla 1. Apartados temáticos destacados en cada grupo de discusión.

Grupo I:
-Dispersión geográfica de los recursos humanos
-Motivación laboral y personal
- Ayuda psico-emocional" para profesionales
-Valoración integral del paciente
-Equidad de los equipos
-Presupuesto social
-Apoyo al cuidador
-Ayuda entre equipos
-Sobrecarga laboral y humana
-Relación con la gerencia
-Futuro de los equipos: "estar quemados"

\section{Grupo II:}

- Atención holística del paciente
- Apoyo psicológico y espiritual
- Trabajo en equipo
- Formación en CP
- Labor de enfermería en CP
- Motivación y sobrecarga laboral
- Protocolo de Acogida en CP
- Cuidar al cuidador-profesional
- Aclaraciones terminológicas o conceptuales

CP, Motivación y sobrecarga laboral del equipo de $\mathrm{CP}$, y Apoyo emocional a los profesionales de CP.

A continuación, se desarrolla cada apartado temático:

\section{- Dispersión geográfica de los recur-} sos humanos: Existe una amplia dispersión geográfica de los recursos humanos, tanto en áreas rurales como urbanas de la $\mathrm{CM}$. Esto conlleva inconvenientes como la cantidad de kilómetros recorridos desde el hospital de referencia hasta el domicilio del paciente (dispersión de hasta $70 \mathrm{~km}$ desde el hospital al domicilio); coste de gasolina, escasamente remunerado en algunos casos; utilización de vehículo pro- pio para tareas de desplazamiento a domicilio; o la sobrecarga del profesional, como consecuencia de todo ello.

- Motivación laboral y personal: A pesar de la mencionada sobrecarga laboral, percibida por la mayoría de los miembros del grupo de discusión, éstos afirman "tener mucha motivación" en sus puestos de trabajo: "No das a basto, prestas tu tiempo, pones tu dinero... porque te gusta mucho tu trabajo". El hecho de que exista un trabajo constante, diario y simultáneo por parte de todos contribuye a que aumente la motivación de las personas que conforman el equipo: "Enseguida se sabe cuando uno (del equipo) no ha intervenido (con el paciente)".Otro aspecto como la docencia 
en el hospital es considerada motivadora para los profesionales, a la vez que supone también una sobrecarga de trabajo, que en ocasiones no se ve reconocido.

- "Ayuda psico-emocional" para profesionales: Se reconoce un déficit importante de recursos "psico-emocionales", pues son pocos los equipos que cuentan con psicólogos y trabajadores sociales. La mayoría de las ocasiones, este apoyo es asumido por profesionales especializados, pero por un periodo de tiempo determinado, a través de obras sociales o, a veces, compartidos con otro centro hospitalario. Hasta hace pocos años la labor psicosocial era desarrollada por el médico y la enfermera encargados del paciente de cuidados paliativos, pudiendo llegar incluso a ser "iatrogénica, pues la formación no se suple con buena voluntad", mencionan.

Aparte, resulta complicado para los profesionales desligar en la práctica la atención psicológica y la espiritual: "La psicóloga (en mi equipo) también ofrece apoyo espiritual. Tenemos claro que es ella la que debe captar esta necesidad; sin embargo, yo a ella también la apoyo cuando es necesario hacer apoyo emocional ", comenta el agente pastoral. Los miembros del grupo opinan que es difícil delimitar quién y cuando hacer atención espiritual; así, apuntan la formación en espiritualidad como un aspecto a incluir en el currículum de los profesionales de CP en beneficio de sus pacientes.

- Valoración integral del paciente: Para los profesionales es necesaria una valoración holística del paciente desde el comienzo. Piensan que un equipo multidisciplinar puede constituir la base de su trabajo, compuesto principalmente por médico, enfermería, atención psicológica, social y espiritual. "Hasta que no hemos contado con trabajador social no hemos tenido conciencia de que hacía falta", comentan, "las familias a veces dan vueltas como peonzas para gestionar un papel".
Además, consideran el apoyo familiar una parte muy importante en el proceso de atención al paciente.

- Equidad de los equipos: Unos centros hospitalarios pueden ofrecer a sus pacientes ciertos recursos técnicos y humanos en calidad y cantidad (programa de duelo, atención telefónica, apoyo en gestiones administrativas, por ejemplo) y otros carecen de ellos. Un ejemplo es el sistema de voluntariado, que funciona sólo en algunos centros. En general, se encuentran lagunas en la atención espiritual, que es bien valorada por los participantes del grupo.

- Presupuesto social: Algunos de los profesionales opinan que, en estos momentos, "el sistema sanitario palia lo que no hay en el sistema social". Se abre un debate en torno al nivel socioeconómico y la preferencia por morir en el domicilio o en el hospital. Los profesionales han observado que, en general, las familias más desfavorecidas socialmente (etnia gitana, inmigrantes, o personas en situación de desempleo) eligen cuidar a su familiar en casa, mientras que las familias que cuentan con mayor nivel económico o tienen horarios de trabajo incompatibles con el cuidado de un enfermo, prefieren que el hospital se haga cargo del paciente. Los profesionales del grupo piensan que la mejor opción para atender a un paciente de paliativos es su domicilio particular, aunque económicamente es costoso de asumir para la familia.

- Apoyo al cuidador: La figura del cuidador es clave para los profesionales. La mayoría de los cuidadores son mujeres de entre 60 y 80 años, con escaso o nulo apoyo familiar, y "es fundamental sentirte apoyado en este proceso", comentan. En algún momento, se produce un agotamiento del cuidador principal y muchos optan por contratar los cuidados de una persona ajena a la familia; sobre todo, se observa con personas de alto nivel cultural; en contrapo- 
sición, cuando el nivel cultural es más bajo, el paciente y sus familiares "prefieren que fallezca en casa" al cuidado de los suyos.

- Ayuda entre equipos: Los profesionales consideran que las personas que trabajan en el área de cuidados Paliativos son "sensibles a lo que les rodea", "a la gente (en este ámbito) le gusta lo que hace", "hay compañerismo". Cuando en una Unidad les falta un recurso, en un momento dado, suelen acudir a un compañero de otra Unidad ubicada en otro centro.

- Sobrecarga laboral y humana: Derivado de la carga laboral, y consecuentemente humana, afloran cuestiones tales como la coordinación entre profesionales, que a veces genera incidencias en la derivación de pacientes, "saltándose" profesionales en el proceso asistencial. Otra preocupación es en el tema de las camas públicas, pues dicen están ubicadas lejos del núcleo urbano y muchos pacientes no quieren trasladarse hasta estos centros de la periferia. Además, la formación de los profesionales españoles en CP es heterogénea, pues no existe una formación reglada con titulación oficial y esto es suplido con master o cursos de diferente índole, "exceptuando el master en Psicooncología". También afecta a la sobrecarga laboral y humana de los profesionales que trabajan con niños el no disponer de hospitales de día al uso, de no contar con mayor número de médicos para guardias, o una atención 24 horas; todo ello, contribuye a que "no se llega por un gran volumen de trabajo". Un último tema que les parece complejo de "enfrentar" en relación a la sobrecarga de trabajo son ciertos pacientes no oncológicos con unas características concretas (por ejemplo, una persona joven con SIDA), pues lleva a los profesionales de CP a cuestionarse si el paciente está en situación de terminalidad; consideran que en ocasiones no saben cómo manejar algunas circunstancias y sienten que el ámbito de $\mathrm{CP}$ se trata como un "cajón de sastre".
- Relación con la gerencia: Los profesionales pertenecientes al ESAPD reconocen tener buena relación con la gerencia. Sin embargo, el Área única hace que otros profesionales se planteen dudas sobre la dependencia de su servicio: "¿De quién depende la toma de decisiones de cada cosa?". Han surgido incidencias respecto a la población rural, por encontrarse más distante de los centros sanitarios de referencia, y se han hecho conscientes de que "en el entorno rural hay tanta demanda de servicios como en el urbano".

- Futuro de los equipos: "estar quemados": La falta o escasez de recursos tanto materiales como humanos hace pensar en el síndrome de burnout a muchos de los participantes. Por otro lado, los asistentes al grupo comparten la importancia que se debe proporcionar al "proceso" en los cuidados paliativos y a la obtención de herramientas adecuadas para prevenir (más en el caso de familiares de niños) o tratar el duelo, puntos clave para no terminar "quemados".

- Trabajo en equipo: El concepto más destacado a lo largo del discurso de los participantes en el grupo ha sido el de Trabajo en equipo: "Lo que ayuda es el trabajo en equipo, lo que hace trabajar con ganas". Entienden que el "peso del trabajo" está distribuido cuando los roles de los componentes del equipo de CP están bien definidos y cada uno trabaja en su disciplina pero de manera conjunta, siendo respetuoso con la labor del compañero. Trabajar en equipo, en muchas ocasiones, implica "reunirnos, reunirnos y reunirnos...", explica un participante, "...es lo más cansado, pero lo más efectivo"; además, este tipo de actuaciones, añade otro, "sirven para abreviar trabajo y responsabilidades".

- Formación en CP: El grupo de discusión hace hincapié en la formación de los profesionales dedicados a los cuidados paliativos; la consideran muy importante para el desempeño de sus tareas y favorece 
otro aspecto destacado anteriormente, la definición de roles, facilitando una mejor labor asistencial y unos resultados eficaces en relación a los pacientes. "Si estas formado se nota,... el profesional sabe hacer bien su trabajo".Como se ha apuntado en un epígrafe anterior, la formación en espiritualidad también es de interés para el equipo de CP.

\section{- Labor de enfermería en Cuidados}

Paliativos: Una de las claves en el ámbito de CP es la atención al paciente por parte de enfermería. Los enfermeros/as deberían estar bien entrenados y contar con unos roles específicos; esta situación llevaría a "descargar mucho trabajo" al equipo. Sin embargo, consideran que este colectivo se encuentra con trabajos precarios, posee falta de formación en CP, acceden a puestos inestables en los centros privados y están a merced de muchas rotaciones. "Se trata de hacer un grupo compacto de enfermería, en el cual se eviten las sustituciones, incluso en verano. Tienes gente entrenada y eso te hace trabajar con facilidad."

- Protocolo de acogida: Un punto en el cual los profesionales se muestran de acuerdo, es el de trabajar inicialmente dentro del marco de un Protocolo de acogida; se trata del primer contacto con el paciente y su familia, bien cuando ingresa en la Unidad de Cuidados Paliativos bien cuando es atendido en el domicilio. Consiste en la presentación de todos los miembros del equipo (médico, persona de enfermería asignada, psicólogo, trabajador social y agente pastoral, si lo hubiera), con una duración aproximada de 15 minutos. Se considera una oportunidad para detectar problemas o explorar de forma somera las necesidades del paciente (mitos, conspiración de silencio, etc.), cada uno desde su ámbito de actuación, a veces incluso con diferentes puntos de vista, pero de manera conjunta. El simple hecho de seguir un "protocolo de bienvenida" parece que facilita considerablemente la relación entre el equipo de CP y el paciente, así como el desempeño laboral de algunos profesionales que encuentran más dificultades a la hora de establecer este primer acercamiento, como puede ser la figura del psicólogo o del agente pastoral.

- Cuidar al profesional: Se entiende por cuidadores en este contexto a los propios profesionales sanitarios que atienden a los pacientes de CP. Solicitan ya sea diferentes herramientas, pautas precisas,...sobre todo en el ámbito de enfermería, pues consideran que actualmente su trabajo ha aumentado de forma considerable. Otros recursos como conductores o número de coches para asistir a los domicilios, siguen siendo deficientes, e incluso a veces son los mismos que cuando se comenzó a trabajar en los ESAD; sin embargo, en estos momentos el número de pacientes es mayor que antes: "Yo me alegro de que sigamos, sobre todo por el beneficio de las familias y los pacientes, pero hay dificultades y seguimos igual".Perciben como un aspecto a favor la presencia de psicólogos en los equipos de cuidados paliativos, pues éstos no solo realizan su labor con los pacientes si no que también pueden contribuir al apoyo emocional de los demás compañeros del equipo.

- Aclaraciones terminológicas o conceptuales: En ocasiones, con las familias surge un debate en torno a la terminología utilizada alrededor de los cuidados paliativos; términos como sedación, sedación paliativa o eutanasia no son siempre bien comprendidos por el enfermo y/o sus familiares. Los profesionales opinan que cuando explican a la familia cómo funciona la sedación aumenta su conocimiento y comprensión sobre el tema. El grupo considera que sería bueno aclarar este tipo de confusiones partiendo muchas veces de los medios de comunicación (radio, televisión, diarios...) y del ámbito político, por ejemplo elaborando un "libro de 
estilo" con terminología de CP: "(se hace necesario) conocer los términos cuando se aplican en la televisión o en otros medios", "somos prisioneros del lenguaje, en los medios escuchas cosas que confunden".

\section{DISCUSIÓN / CONCLUSIONES}

Con los resultados reflejados en este trabajo, el objetivo último del proyecto es construir un Cuestionario de Recursos para la Provisión de Servicios en Cuidados Paliativos, presentado en el apartado de Antecedentes, como una herramienta válida para el uso de los profesionales en el ámbito de CP en la CM y fuera de ella. Para esto, se llevaría a cabo como fase final un proceso de evaluación de validez y fiabilidad a través del pilotaje del cuestionario por distintos centros asistenciales.

Posteriormente, los resultados de este trabajo podrán ser utilizados como fuente de información para la modificación del cuestionario de recursos en Cuidados Paliativos y para un proyecto sobre el rediseño del mapa de procesos del flujo asistencial en CP de la CM y en otros entornos sanitarios con este tipo de servicios.

Algunas limitaciones en este trabajo han sido por una parte, el no poder incorporar a los grupos de discusión otro tipo de perfiles profesionales como farmacéuticos, fisioterapeutas, rehabilitadores o terapeutas ocupacionales. Si bien es cierto que en nuestro contexto no es habitual contar con ellos, en otros países se consideran miembros integrantes del equipo de Cuidados Paliativos.

Por otro lado, en una etapa anterior del proyecto, como se comenta al inicio de este artículo, se contestaron un total de 40 cuestionarios por parte de profesionales específicos de las unidades de CP de la CM. En estos cuestionarios, encontramos muchos de los ítems sin rellenar, lo que limitó el análisis descriptivo de los mismos; otros, estaban completados con diferentes parámetros (números netos, porcentajes) lo que igualmente limitó su análisis. Sin embargo, este aspecto queda solventado cuando mediante los grupos de discusión establecidos se les preguntó a los profesionales en base a su experiencia sobre el nivel de concordancia con la realidad de su trabajo, grado de complejidad y dudas al completar el cuestionario de recursos, preguntas ambiguas, adecuación a los recursos que les rodean y valoración de los ítems abordados en el cuestionario.

De la revisión sistemática que se elaboró conjuntamente con este trabajo cualitativo, se desprende, entre otros aspectos, que existen diferentes cuestionarios que pretenden medir las relaciones y las percepciones de los miembros del equipo de CP. Siempre para mejorar la calidad y la eficacia en la atención al paciente al final de la vida. La mayoría de los estudios están basados en los facilitadores y barreras para el trabajo en un equipo multidisciplinar $^{(4-12)}$. Estos equipos son la base de la atención paliativa, donde el éxito o el fracaso de la calidad de la atención, depende de la integración, filosofía, autonomía y confianza mutua de los integrantes del mismo.

Otras cuestiones medidas son los roles y competencias de cada uno de los miembros del equipo. El compromiso del trabajo en equipo y tener como objetivo la mejor atención del paciente, puede ser clave en el mejor aprovechamiento de las funciones de los integrantes del equipo multidisciplinar.

Por último, existe una tendencia en la literatura de los últimos años, en intentar definir en el paciente con necesidades paliativas, el perfil de los profesionales generalistas frente a los expertos ${ }^{(13)}$. No están siempre claros los puntos en común y los que marcan la diferencia entre ambos. Sin embargo, es fundamental, diferenciar $y$ determinar claramente el perfil de ambos tipos de profesionales, para mejorar la ca- 
lidad de la atención e intentar conseguir la excelencia en los cuidados. En ocasiones, una súper-especialización puede ser un problema y ser una barrera para el trabajo en equipo.

Este trabajo pretende ser el punto de partida para la elaboración y validación de un cuestionario en la provisión de servicios en Cuidados Paliativos, y así poder evaluar los recursos humanos y técnicos de nuestra red asistencial sanitaria paliativa.

\section{REFERENCIAS BIBLIOGRÁFICAS}

1. Ruiz López D; Izquierdo Botica F; Guerra Rodríguez M; García-Baquero Merino MT; Blasco Amaro JA. Validación y Evaluación de los Resultados del Cuestionario de Recursos para la provisión de Servicios en Cuidados Paliativos en la Comunidad de Madrid. Unidad de Evaluación de Tecnologías Sanitarias (UETS) de la Agencia Laín Entralgo, y Coordinación Regional de Cuidados Paliativos. Consejería de Sanidad, Comunidad de Madrid, 2011.

2. Consejería de Sanidad y Consumo. Comunidad de Madrid. Plan Estratégico de Cuidados Paliativos de la Comunidad de Madrid 2010-2014.

3. Valles MS. Entrevistas cualitativas. Colección Cuadernos Metodológicos, NúM. 32. Madrid: Centro de Investigaciones Sociológicas; 2009.

4. Guyatt GH, Sackett DL, Cook DJ, Cook DJ, Guyatt G, Bass E. et al. Users' guides to the medical literature. II. How to use an article about therapy or prevention. Are the results of the study valid? JAMA 1993;270:2598-601. Doi:10.1001/ jama.1993.03510210084032.

5. Salomon L, Belouet C, Vinant-Binam P, Sicard Didier, Vidal-Trecan G. A terminal care support team in a Paris University
Hospital: Care providers' views. J Palliat Care 2001;17:109-16.

6. Tolson D, McIntosh J, Loftus L, Cormie P. Developing a managed clinical network in palliative care: A realistic evaluation. Int J Nurs Stud 2007;44:183-95. Doi: 10.1016/j.ijnurstu.2005.11.027

7. Jünger $S$, Pestinger $M$, Elsner $F, N$ Krumm N, Radbruch L. Criteria for successful multiprofessional cooperation in palliative care teams. Palliat Med 2007;21:347-54. Doi: 10.1177/0269216307078505

8. Shipman C, Gysels M, White P, Worth A, Murray SA, Barclay $S$ et al. Improving generalist end of life care: National consultation with practitioners, commissioners, academics, and service user groups. BMJ 2008;337:a1720. Doi: 10.1136/bmj.a1720.

9. Gómez-Batiste X, Nabal Vicuña M, Trelis Navarro J, Roca Casas J, Porta Sales J. Paz Ruiz S, Espinosa Rojas J. Evaluación cualitativa del desarrollo del Proyecto Demostrativo OMS de Cuidados Paliativos de Cataluña a los 15 años de su implementación y propuestas de mejora del programa. Med Paliat 2008;15:293-300.

10. Oliver DP, Wittenberg-Lyles EM, Day M. Measuring interdisciplinary perceptions of collaboration on hospice teams. Am J Hosp Palliat Care 2007;24:49-53. Doi: 10.1177/1049909106295283

11. O'Connor M, Fisher C, Guilfoyle A. Interdisciplinary teams in palliative care: A critical reflection. Int J Palliat Nurs 2006;12:132-7.

12. Oliver DP, Wittenberg-Lyles EM, Day M. Variances in perceptions of interdisciplinary collaboration by hospice staff. J PaIliat Care 2006;22:275-80.

13. Spruyt O. Team networking in palliative care. Indian J Palliat Care (Suppl) 2011:S17-S19. Doi: 10.4103/09731075.76234 
